Introduction: Properly planned and performed diagnostic tests allow the optimal treatment option to be chosen for the patient. They also allow qualification for the correct surgical procedure.

Aim of the study: In this study we evaluated the clinical value of preoperative ultrasound scan (USS) testing performed during primary disease staging in patients with early breast cancer qualified to sentinel lymph node biopsy (SLNB).

Material and methods: The group of breast cancer patients who underwent SLNB from March 2012 to May 2013. As well as the standard procedure of the preoperative diagnostics model, in each patient the USS of axillary lymph nodes was performed additionally. The results were compared with the data from postoperative pathological reports. We attempted to define the factors influencing the possibility of obtaining false positive and false negative USS results.

Results: The analysis comprised 172 patients. In $14.4 \%$ of cases with normal USS result the pathological result was different from the expected one (pN1). In $42.3 \%$ of patients with suspicious axillary lymph nodes the result of the pathological report was positive. The sensitivity of the USS testing was $89.3 \%$, and the specificity was $34.4 \%$, PPV $-85.6 \%$, NPV $-42.3 \%$

Conclusions: Ultrasonographic assessment of axillary lymph nodes in breast cancer patients qualified for SLNB is a test with high sensitivity and high predictive value of the positive test result. The possibility of a result contrary to the actual nodal status may result primarily from the technical limitations of USS testing.

Key words: breast cancer, ultrasonog raphy, sensitivity, specificity, conserving treatment, sentinel node.

Contemp Oncol (Pozn) 2018; 22 (4): 247-251 DOI: https://doi.org/10.5114/wo.2018.82644

\section{Analysis of the causes of false negative and false positive results of preoperative axillary ultrasound in patients with early breast cancer - a single-centre study}

\author{
Tomasz Nowikiewicz ${ }^{1,2}$, Adam Nowak ${ }^{3}$, Magdalena Wiśniewska ${ }^{4,5}$, \\ Michał Wiśniewski ${ }^{6}$, Magdalena Nowikiewicz ${ }^{7}$, Wojciech Zegarski ${ }^{1,2}$
}

\begin{abstract}
${ }^{1}$ Chair and Department of Surgical Oncology, Ludwik Rydygier's Collegium Medicum in Bydgoszcz, Nicolaus Copernicus University in Torun, Bydgoszcz, Poland ${ }^{2}$ Department of Breast Cancer and Reconstructive Surgery, Prof. Franciszek Łukaszczyk Oncology Centre, Bydgoszcz, Poland

${ }^{3}$ Diagnostic Imaging and Interventional Radiology Unit, Prof. Franciszek Łukaszczyk Oncology Centre, Bydgoszcz, Poland

${ }^{4}$ Department of Oncology and Brachytherapy, Ludwik Rydygier's Collegium Medicum in Bydgoszcz, Nicolaus Copernicus University in Torun, Bydgoszcz, Poland ${ }^{5}$ Chemotherapy Department, Prof. Franciszek Łukaszczyk Oncology Centre, Bydgoszcz, Poland

${ }^{6}$ Outpatient Chemotherapy Department, Prof. Franciszek Łukaszczyk Oncology Centre, Bydgoszcz, Poland

"Student Scientific Society, Chair and Department of Surgical Oncology, Ludwik Rydygier's Collegium Medicum in Bydgoszcz, Nicolaus Copernicus University in Torun, Bydgoszcz, Poland
\end{abstract}

\section{Introduction}

Breast cancer is the most common cause of death in women in developed countries. The increase in the number of newly diagnosed cases of the disease is accompanied by a decreased mortality rate. This trend is observed particularly in countries of Western Europe and the United States of America [1].

The prognostic data describing the epidemiological situation in Poland show that at the end of the current decade the number of new breast cancer cases may exceed 20,000 a year [1, 2]. According to information from the National Cancer Registry in Poland there is little increase in the five-year relative survival rate: from $75 \%$ in $2000-2002$ to $77.2 \%$ in $2003-2005$ [3].

Alongside the gradually increasing number of newly diagnosed breast cancer cases in Poland, the overall rate of early-stage breast cancer has also increased. This phenomenon resulted from an organised, population-based mammography screening program [4]. Diagnosing breast cancer quickly and accurately is vital because the earlier it is detected, the greater the chances of implementing conserving procedures - sentinel lymph node biopsy (SLNB) and breast conserving therapy $(\mathrm{BCT})[3,4]$.

Properly planned and performed diagnostic tests allow reliable determination of the clinical stage of cancer. They also make it possible to choose the right treatment strategy for the patient. According to the current clinical standards, the necessary tasks regarding therapeutic management of breast cancer patients also include the retrospective audits related to the overall treatment outcomes $[5,6]$. This also applies to determining the type of operating procedure needed, which is particularly important for patients with breast cancer. As demonstrated by Gruber et al., properly performed preoperative diagnostics reduces the incidence of non-therapeutic axillary lymphadenectomy by $40 \%$ [7].

Among currently used diagnostic methods for axillary lymph nodes verification in patients with invasive breast cancer, ultrasonography (USS) is the 
most accessible and affordable. It is also characterised by reproducibility and possibility of high objectification of obtained results [8]. The sensitivity and specificity of this test in assessing the presence of metastatic lesions in regional lymph nodes is $53-96 \%$ and $50-75 \%$, respectively [9-12].

In addition to the above-mentioned advantages, USS scans make it possible to increase the sensitivity of screening for early detection of breast cancer, routinely performed mammography [13]. This is especially noticeable in patients with significant breast density, including the presence of suspicious microcalcifications [13-15]. Postoperative ultrasonography surveillance is feasible in breast cancer patients, especially following conserving procedures (SLNB, BCT) $[13,16]$.

In this study, we evaluated the clinical value of preoperative USS performed during initial staging of the disease in patients with early-stage breast cancer. The analysis focused mainly on the possibility of USS examination for accurate verification of axillary lymph nodes.

\section{Material and methods}

The study was conducted in the form of a prospective analysis. The evaluation included patients with locally advanced breast cancer, who were referred for the sentinel node biopsy procedure. Patients were hospitalised in the period from March 2012 to May 2013, in the highest reference centre for surgical treatment of malignant neoplasms of the breast.

Patients enrolled to the trial did not have suspected axillary lymph nodes (patients with NO score of clinical staging) in physical examination and USS at cancer diagnosis. Another criterion was a period of eight weeks prior to the surgery, during which initial USS examination was performed.

One day prior to the procedure, an additional assessment of the axillary lymph nodes was performed in each patient. All tests were conducted by the same radiologist. Regardless of the possible findings in the study of abnormalities within the armpit, each patient underwent a sentinel node biopsy (Fig. 1).

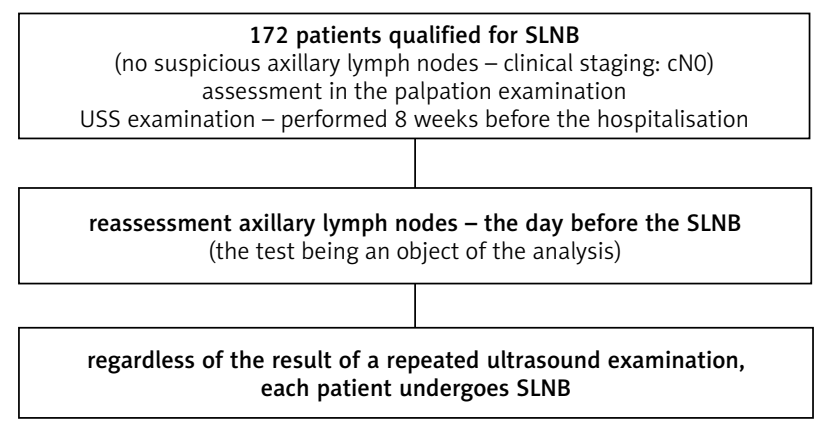

SLNB - sentinel lymph node biopsy; CNO - patients without metastases in the axillary lymph nodes: the preoperative clinical assessment included a physical examination and an axillary ultrasound; USS - ultrasonography scan

Fig. 1. Study qualification scheme
After assessing the results of the pathological report, the usefulness of preoperative ultrasonography for the detection of metastatic lesions present in SLNB-removed lymph nodes was verified. The sensitivity and specificity of the test and the predictive value of the positive and negative test results were determined for this purpose. Definitions: test sensitivity - the quotient of the results of the truly positive test and the sum of the results of the truly positive and the false negative: TP/TP + FN (true positive, false negative), test specificity - the quotient of the results truly negative and the sum of the results truly negative and false positive: TN/TN + FP (true negative, false positive), positive predictive value (PPV) - the quotient of the results of the truly positive test and the sum of the results truly positive and false positive (TP/TP + FP), and negative predictive value (NPV) - the quotient of the results truly negative and the sum of the results truly negative and false negative (TN/TN + FN).

An attempt was also made to define factors that may affect the onset of abnormal preoperative ultrasonography (false-positive and false-negative results). The analysis included the clinical data of patients (age, BMI) and clinical features of breast cancer (tumour size - clinical and pathological findings, palpation of lesions, histological grade, histological type and cancer type, oestrogen, progesterone, and HER2 receptor status, and Ki-67 mitotic factor value). For statistical calculations, a linear regression model was used. The occurrence of a statistically significant difference between the compared groups of data was recognised at the values of significance level $p<0.05$.

\section{Results}

The analysis comprised 172 patients aged $59.6 \pm 10.2$ years (range 32 to 81 years).

In nearly $85 \%$ of patients, preoperative assessment of axillary lymph nodes showed no suspicious lesions. 26 patients (15.1\%) were diagnosed with questionable lymph nodes (Fig. 2).

As a result of the pathological assessment of lymph nodes removed during SLNB, 32 patients (18.6\%) were diagnosed with metastatic lesions. In the case of $14.4 \%$

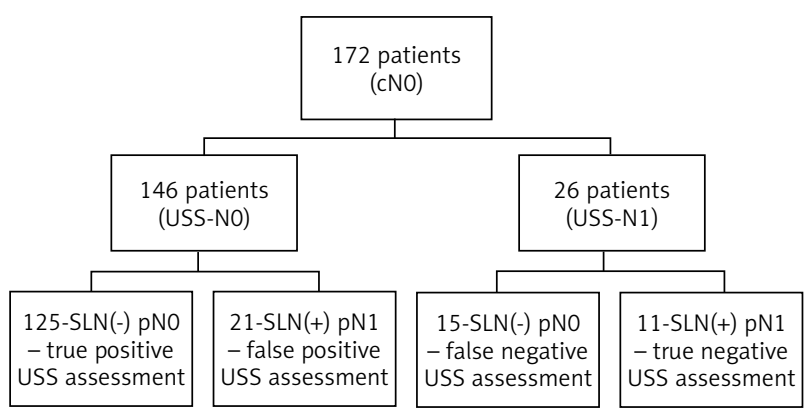

Fig. 2. Results of preoperative USS examination of axillary lymph nodes and pathological assessment of sentinel nodes removed during SLNB 
of patients (21/146) with correct axillary ultrasonography (USS - NO patients), prognostic testing was not confirmed (histopathological result - pN1, patients with false positive USS scan). In $42.3 \%$ of patients (11/26) presenting suspected lymph nodes in USS examination - N1 patients histopathological findings from SLNB revealed metastatic lesions (with a truly positive result of USS, Fig. 2). The sensitivity of preoperative ultrasonography scan was $89.3 \%$, specificity $34.4 \%$, PPV $-85.6 \%$, and NPV $-42.3 \%$.

Based on the performed calculations, none of the clinical factors tested showed a statistically significant effect on the possibility of abnormal ultrasonography result. A detailed summary of the clinical data of patients with normal (positive and negative) and abnormal (false positive and false negative) results from the preoperative evaluation of axillary lymph nodes is presented in Table 1.

\section{Discussion}

Consistent with the trial's aim, selective assessment of the diagnostic value of ultrasonography in breast cancer patients qualified to procedures conserving lymph nodes of the axillary fossa was conducted. Unlike most clinical trials, verified imaging studies were performed directly prior to surgical procedure, regardless of former preoperative diagnostics. In this way, the possibility of influencing the result of the length of time between the time of performing the test and performing the surgery is omitted. It brings new perspectives to preoperative diagnostics of patients submitted for SLNB procedure.

According to our previous analyses, the lowest rates of metastasis in sentinel lymph nodes can be found in patients who have undergone a USS scan (with correct results) directly prior to SLNB procedure. This concerns the sensitivity as well as the specificity of the study being evaluated [16].

We also resigned from the additional methods for verifying axillary lymph nodes, as shown by some of the ultrasound examinations we reviewed. According to the accepted research criteria, a prospective analysis focused on the selective evaluation of the clinical value of USS testing. Therefore, in spite of the preoperative axillary lymph node abnormalities, each patient underwent sentinel node biopsy. This proceeding was consistent with initial qualification for SLNB and corresponded with the standards for the diagnosis and treatment of early-stage breast cancer (SLNB was performed in patients with cNO - clinical assessment of axillary lymph node status).

Ultrasound was performed by one radiologist using the same ultrasound device, in order to achieve objective results.

Therefore, the principles adopted in our study may not correspond to the standard preparation for surgical procedure of patients with early-stage breast cancer. However, they make it possible to significantly reduce the impact on the results of other factors, apart from the factors selected for evaluation in a planned way.

According to Pinheiro et al., axillary USS scan should be an integral part of routine diagnostic practice in patients with breast cancer. This is especially important for patients with primary tumours greater than $1 \mathrm{~cm}$, because patients in this group benefit most from such procedures [17].

An analysis of the clinical value of preoperative USS scan was performed by Perhavec et al. The study included 470 breast cancer patients enrolled to the sentinel node biopsy procedure. The rate of metastatic lesions found after the SLNB was 43\%. For patients diagnosed with preoperative ultrasonography, the percentage was lower - 39\% (101/257), and in patients in whom only physical examination was performed before SLNB - 47\% (101/213; $p=0.092$ ). Patients in the USS group had a lower proportion of macrometastases in the removed sentinel lymph nodes (44\% vs. 66\%; $p=0.002)$. These changes were also smaller in size (5.4 vs. $6.8 \mathrm{~mm} ; p=0.027$ ). According to the authors of the cited article, a higher diagnostic value in determining the preoperative status of the axillary lymph nodes is verified by ultrasonography (false-negative results in $39 \%$ of patients). On the other hand, the limitation of the preoperative assessment of axillary lymph node status for physical examination resulted in the presence of metastatic lesions in $47 \%$ of patients in a histopathological report of sentinel lymph nodes [18].

In a Johnson et al. study of 155 patients with normal preoperative ultrasonography, $29 \%$ had a positive histopathological examination after lymphadenectomy. In the group of patients with negative lymph node ultrasonography, tumour size was significantly lower (21 vs. 30 mm; $p<0.02)$. In patients with false positive USS scan there were more cases of invasive lobular cancer (5\% vs. 13\%; $p<0.001)$ and more cases of lymph vessel invasion (5\% vs. 31\%; $p<0.001$ ) [19]. In contrast to the cited study, there were no statistically significant differences in the patients we analysed for any of the clinical and pathological data included in the calculation.

In the Diepstraten et al. study of 9212 patients, it was demonstrated that up to $25 \%$ of correct USS results performed prior to SLNB were false negative. According to the authors, the clinical value of preoperative USS scan can be augmented by fine-needle aspiration biopsy (FNAB) of lymph nodes in carefully selected patients. It has been shown that the combination of both diagnostic methods enables detection of metastases in axillary lymph nodes in $50 \%$ of patients with false-negative lymph nodes evaluated in the USS test [20]. In Baruah et al.'s study preoperative USS scan combined with the aspiration biopsy of suspected lymph nodes allowed the avoidance of unnecessary SLNB in $28.5 \%$ of patients (in the group of patients with cancer cells as a result of biopsy, qualified for axillary lymphadenectomy with positive results of histological examination) [21].

An attempt was made to compare the treatment outcomes of patients with invasive breast cancer, who were enrolled in the sentinel node biopsy or treated without axillary lymph node verification in the European Institute of Oncology in Milan, in the ongoing SOUND (sentinel node vs. observation after axillary ultrasound) study. Clinical trial patient inclusion criteria are either the correct outcome of preoperative axillary lymph node USS scan, or the presence of a single positive lymph node verified in preoperative FNAB. The expected outcome of the study is the lack of statistical- 
Table 1. Study patients - clinical and pathological features

\begin{tabular}{|c|c|c|c|c|c|}
\hline \multirow[t]{2}{*}{ Clinical and pathological features } & \multicolumn{2}{|c|}{$\begin{array}{l}\text { Correct result of preoperative USS } \\
\qquad \begin{array}{c}(\mathrm{TN}+\mathrm{TP}) \\
n=136\end{array}\end{array}$} & \multicolumn{2}{|c|}{$\begin{array}{l}\text { Incorrect result of preoperative USS } \\
\qquad \begin{array}{c}\text { (FN }+ \text { FP) } \\
n=36 \\
\end{array}\end{array}$} & \multirow[t]{2}{*}{$p$} \\
\hline & $n$ & $\%$ & $n$ & $\%$ & \\
\hline \multicolumn{6}{|l|}{ Age } \\
\hline$<40$ years & 9 & 6.6 & 2 & 5.6 & 0.896 \\
\hline 40 to 60 years & 63 & 46.3 & 13 & 36.1 & 0.765 \\
\hline$>60$ years & 64 & 47.1 & 21 & 58.3 & 0.657 \\
\hline \multicolumn{6}{|l|}{ BMI } \\
\hline Normal (18.5-24.9) & 47 & 34.6 & 12 & 33.3 & 0.876 \\
\hline Overweight (25-29.9) & 52 & 38.2 & 13 & 36.1 & 0.764 \\
\hline Obesity $(\geq 30)$ & 37 & 27.2 & 11 & 30.6 & 0.795 \\
\hline Palpable tumour & 79 & 58.1 & 24 & 66.7 & 0.205 \\
\hline \multicolumn{6}{|l|}{ Tumour size (in USS scan) } \\
\hline T1a & 14 & 10.7 & - & - & - \\
\hline $\mathrm{T} 1 \mathrm{~b}$ & 37 & 28.2 & 7 & 19.4 & 0.322 \\
\hline $\mathrm{T} 1 \mathrm{c}$ & 66 & 50.4 & 21 & 58.3 & 0.687 \\
\hline $\mathrm{T} 2$ & 14 & 10.7 & 8 & 22.2 & 0.778 \\
\hline T3 & - & - & - & - & - \\
\hline No data & 5 & - & - & - & - \\
\hline \multicolumn{6}{|c|}{ Tumour size (pathological report - pT) } \\
\hline TO (after BMU) & 2 & 1.5 & - & - & - \\
\hline T1mic & 4 & 3.0 & 1 & 2.8 & 0.288 \\
\hline $\mathrm{T} 1 \mathrm{a}$ & 3 & 2.2 & - & - & - \\
\hline $\mathrm{T} 1 \mathrm{~b}$ & 23 & 17.0 & 3 & 8.3 & 0.657 \\
\hline $\mathrm{T} 1 \mathrm{c}$ & 77 & 57.0 & 19 & 52.8 & 0.864 \\
\hline $\mathrm{T} 2$ & 26 & 19.3 & 13 & 36.1 & 0.764 \\
\hline T3 & - & - & - & - & - \\
\hline T4 & 1 & 0.6 & - & - & - \\
\hline \multicolumn{6}{|l|}{ Histological grading } \\
\hline G1 & 12 & 9.2 & 3 & 8.6 & 0.674 \\
\hline G2 & 95 & 72.5 & 24 & 68.6 & 0.453 \\
\hline G3 & 24 & 18.3 & 8 & 22.9 & 0.452 \\
\hline no data & 5 & - & 1 & - & - \\
\hline \multicolumn{6}{|l|}{ Histological type } \\
\hline ductal carcinoma & 115 & 84.6 & 32 & 88.9 & 0.673 \\
\hline lobular carcinoma & 18 & 13.2 & 3 & 8.3 & 0.564 \\
\hline another invasive type & 3 & 2.2 & 1 & 2.8 & 0.765 \\
\hline ER positive & 116 & 85.3 & 28 & 77.8 & 0.345 \\
\hline ER negative & 20 & 14.7 & 8 & 22.2 & 0.632 \\
\hline PR positive & 116 & 85.3 & 28 & 77.8 & 0.433 \\
\hline PR negative & 20 & 14.7 & 8 & 22.2 & 0.245 \\
\hline HER2 positive & 19 & 14.0 & 2 & 5.6 & 0.236 \\
\hline HER2 negative & 117 & 86.0 & 34 & 94.4 & 0.435 \\
\hline \multicolumn{6}{|l|}{ Ki-67 index } \\
\hline $0-14 \%$ & 37 & 38.1 & 17 & 60.7 & 0.534 \\
\hline $15-30 \%$ & 49 & 50.5 & 6 & 21.4 & 0.653 \\
\hline $31-45 \%$ & 4 & 4.1 & 4 & 14.3 & 0.247 \\
\hline above $45 \%$ & 7 & 7.2 & 1 & 3.6 & 0.543 \\
\hline no data & 39 & - & 8 & - & - \\
\hline \multicolumn{6}{|l|}{ Biological type } \\
\hline luminal A & 37 & 30.3 & 17 & 50.0 & 0.534 \\
\hline luminal B HER2 negative & 56 & 45.9 & 8 & 23.5 & 0.653 \\
\hline luminal B HER2 positive & 10 & 8.2 & 1 & 2.9 & 0.435 \\
\hline HER2 positive & 9 & 7.4 & 1 & 2.9 & 0.678 \\
\hline triple negative & 10 & 8.2 & 7 & 20.6 & 0.634 \\
\hline no data & 14 & - & 2 & - & - \\
\hline All & 136 & 100 & 36 & 100 & \\
\hline
\end{tabular}

$T N$ - negative result; TP - positive result; $F N$ - false negative result; FP - false positive result; $n$ - number of patients; $p$ - significance level; BMI - body mass index; $B M U$ - Mammotome biopsy; $E R$ - oestrogen receptor; $P R$ - progesterone receptor; HER2 - HER2 receptor 
ly significant differences in the long-term follow-up in both groups of patients, which may allow a change in the standard of care for patients with early-stage breast cancer [22].

The sensitivity of preoperative ultrasonography in detecting metastatic lesions in sentinel nodes in the study group was $89.3 \%$. This is comparable to the results acquired by Ciatto et al. (53-96\%) and other authors [9-12]. In Koehler et al., Genty et al., and Garcia-Ortega et al., the specificity of USS assessment was 50-75\% [9, 11, 12]. Combined use of ultrasound with a FNAB is a useful complementary tool improving specificity of the verification to $100 \%$ [10]. The value we received at $34.4 \%$ is therefore lower than in the studies mentioned above.

\section{Conclusions}

As shown in the analysis, it is possible to select patients who are not expected to have metastatic lesions in the lymph nodes removed during the SLNB procedure. This is possible by performing a preoperative USS assessment of axillary lymph nodes in patients with breast cancer, who are referred for treatment conserving lymph nodes of the armpit. Such early diagnostics is a safe and effective way of verifying the nodal status of the axilla.

The ultrasound examination is characterised by high sensitivity and high predictive value of the positive test result. As demonstrated, none of the analysed clinical features contributed to obtaining inaccurate preoperative ultrasound results. It should also be emphasised that technical limitations of the USS examination create restrictions for the study itself because the risk of receiving a non-conforming result (FN, FP results) can arise.

\section{The authors declare no conflict of interest.}

\section{References}

1. Didkowska J, Wojciechowska U. Nowotwory piersi w Polsce i Europie - populacyjny punkt widzenia. Nowotwory J Oncol 2013; 63: 111-118.

2. Wojtyś P, Godlewski D, Antczak A. Predictions of cancer incidence in Poland in 2019. Cent Eur J Med 2013; 8: 185-191.

3. Herman K, Śliwczyński A, Wysocki W. Wyniki, metody i koszty leczenia raka piersi w Polsce (w latach 2005-2007). Nowotwory J Oncol 2014; 64: 33-39.

4. Nowikiewicz T, Zegarski W, Piatkowska M, Klag M. Evaluation of the effects of mammography screening program on cancer progression and implemented treatment in patients with breast cancer. Pol Przegl Chir 2013; 85: 204-209.

5. Głowacka I, Nowikiewicz T, Hagner W, Nowacka K, Sowa M, Zegarski W. Sagittal Plane Postural Changes in Female Patients with Breast Cancer after Different Surgical Techniques. Breast J 2017; 23: 109-111.

6. Głowacka-Mrotek I, Sowa M, Siedlecki Z, Nowikiewicz T, Zegarski W. Evaluation of changes to foot shape in females 5 years after mastectomy - case-control study. Breast Cancer Res Treat 2017; 163: 287-294.

7. Gruber I, Hahn M, Fehm T, Hann von Weyhern C, Stäbler A, Winckelmann A, Wallwiener D, Kühn T. Relevance and methods of interventional breast sonography in preoperative axillary lymph node staging. Ultraschall Med 2012; 33: 337-343.

8. Lee MC, Joh JE, Chau A. Axillary staging prior to neoadjuvant chemotherapy: the roles of sentinel lymph node biopsy and axillary ultrasonography. Cancer Control 2012; 19: 277-285.
9. Koehler KE, Ohlinger R. Sensitivity and specificity of preoperative ultrasonography for diagnosing nodal metastases in patients with breast cancer. Ultraschall Med 2011; 32: 393-399.

10. Ciatto S, Brancato B, Risso G, Ambrogetti D, Bulgaresi P, Maddau C, Turco P, Houssami N. Accuracy of fine needle aspiration cytology (FNAC) of axillary lymph nodes as a triage test in breast cancer staging. Breast Cancer Res Treat 2007; 103: 85-91.

11. Genta F, Zanon E, Camanni M, Deltetto F, Drogo M, Gallo R, Gilardi C. Cost/accuracy ratio analysis in breast cancer patients undergoing ultrasound-guided fine-needle aspiration cytology, sentinel node biopsy, and frozen section of node. World J Surg 2007; 31: 11551163.

12. Garcia-Ortega MJ, Benito MA, Vahamonde EF, Torres PR, Velasco $A B$, Paredes MM. Pretreatment axillary ultrasonography and core biopsy in patients with suspected breast cancer: diagnostic accuracy and impact on management. Eur J Radiol 2011; 79: 64-72.

13. Moon HJ, Jung I, Park SJ, Kim MJ, Youk JH, Kim EK. Comparison of Cancer Yields and Diagnostic Performance of Screening Mammography vs. Supplemental Screening Ultrasound in 4394 Women with Average Risk for Breast Cancer. Ultraschall Med 2015; 36: 255-263.

14. Keränen AK, Haapea M, Rissanen T. Ultrasonography as a Guiding Method in Breast Micro-Calcification Vacuum-Assisted Biopsies. Ultraschall Med 2016; 37: 497-502.

15. Mueller-Schimpfle MP, Brandenbusch VC, Degenhardt F, Duda V, Madjar H, Mundinger A, Rathmann R, Hahn M. The Problem of Mammographic Breast Density - The Position of the DEGUM Working Group on Breast Ultrasound. Ultraschall Med 2016; 37: 170-175.

16. Nowikiewicz T, Nowak A, Wisniewska M, Wiśniewski M, Zegarski W. Diagnostic value of preoperative axillary lymph node ultrasound assessment in patients with breast cancer qualified for sentinel lymph node biopsy. Wideochir Inne Tech Maloinwazyjne 2015; 10: 170-177.

17. Pinheiro DJ, Elias S, Nazario AC. Axillary lymph nodes in breast cancer patients: sonographic evaluation. Radiol Bras 2014; 47: 240-244.

18. Perhavec A, Besic N, Hocevar M, Zgajnar J. Touch imprint cytology of the sentinel lymph nodes might not be indicated in early breast cancer patients with ultrasonically uninvolved axillary lymph nodes. Ann Surg Oncol 2008; 15: 2257-2262.

19. Johnson S, Brown S, Porter G, Steel J, Paisley K, Watkins R, Holgate C. Staging primary breast cancer. Are there tumour pathological features that correlate with a false-negative axillary ultrasound? Clin Radiol 2011; 66: 497-499.

20. Diepstraten SC, Sever AR, Buckens CF, Veldhuis WB, van Dalen T, van den Bosch MA, Mali WP, Verkooijen HM. Value of preoperative ultrasound-guided axillary lymph node biopsy for preventing completion axillary lymph node dissection in breast cancer: a systematic review and meta-analysis. Ann Surg Oncol 2014; 21: 51-59.

21. Baruah BP, Goyal A, Young P, Douglas-Jones AG, Mansel RE. Axillary node staging by ultrasonography and fine-needle aspiration cytology in patients with breast cancer. Br J Surg 2010; 97: 680-683.

22. Gentilini O, Veronesi U. Abandoning sentinel lymph node biopsy in early breast cancer? A new trial in progress at the European Institute of Oncology of Milan (SOUND: Sentinel node vs Observation after axillary UltraSouND). Breast 2012; 21: 678-681.

\section{Address for correspondence}

\section{Tomasz Nowikiewicz}

Department of Breast Cancer and Reconstructive Surgery

Prof. Franciszek Łukaszczyk Oncology Centre

2 Izabeli Romanowskiej St.

85-796 Bydgoszcz, Poland

e-mail: tomasz.nowikiewicz@gmail.com

Submitted: 19.10 .2018

Accepted: $\quad 30.11 .2018$ 